\title{
Infrared observations of AGN
}

\author{
P. Lira ${ }^{1}$, F. Marín ${ }^{1}$, L. Videla ${ }^{1}$, \\ A. Alonso-Herrero ${ }^{2}$, D. M. Alexander ${ }^{3}$ and M. Ward ${ }^{4}$ \\ ${ }^{1}$ Universidad de Chile, Casilla 36-D, Santiago, Chile \\ ${ }^{2}$ DAMIR - IEM, C. Serrano 113-121, 28006 Madrid, Spain \\ ${ }^{3}$ Cambridge University, Madingley Road, Cambridge CB3 0HA, UK \\ ${ }^{4}$ Leicester University, Leicester LE1 7RH, UK
}

\begin{abstract}
We present results from an imaging and spectroscopic study of the dust properties of Seyfert galaxies in the $1-10 \mu \mathrm{m}$ range. The data are compared to state of the art models of torus emission to constrain geometrical and physical properties of the obscuring medium.
\end{abstract}

\section{Introduction}

A key ingredient of the Unified Model for AGN is an axially symmetric dusty structure (a.k.a., the torus) that absorbs UV photons from the central source and re-emits them anisotropically as thermal radiation at near and mid-IR wavelengths. This wavelength range is, therefore, crucial to determine the properties of the obscuring material.

Here we present 1-10 $\mu \mathrm{m}$ nuclear SEDs and N-band spectroscopy for a small sample of Seyfert galaxies. This is part of a much larger study of Seyfert galaxies drawn from the $12 \mu \mathrm{m}$ Extended Galaxy Sample (Rush, Malkan \& Spinoglio 1993) for which we are collecting JHKLMN-band imaging in order to produce nuclear SEDs. The results will be compared with theoretical models of the dusty torus (Nenkova, Ivezić \& Elitzur 2002), $\mathrm{N}_{\mathrm{H}}$ columns obtained from X-ray observations (Bassani et al. 1999), and spectropolarimetry (Tran 2003). We expect to put statistically significant constraints on the physical and geometrical characteristics of the obscuring material around AGN.

\section{Observations, data analysis and future work}

We have obtained UKIRT/Michelle N-band spectroscopy for 4 Seyfert 2s (S2s: Mk509, Mk1066, NGC1068, NGC7674) and 2 Seyfert 1 galaxies (S1s: MCG+8-11-11, NGC7469). Using a PAH dominated template from starburst emission (kindly provided by E. Le Floc'h) we have estimated the contribution from circumnuclear star-forming regions to the observed spectra. We find that only for Mk1066 the starburst contribution is significant $(\sim 50 \%)$, being typically $\lesssim 10 \%$ for all other nuclei (Fig. 1). Isolating the AGN continuum is crucial when trying to determine the profile of the $9.7 \mu \mathrm{m}$ dust feature, which can be very useful as a diagnostic for dust conditions (Nenkova, Ivezić \& Elitzur 2002), since the starburst related PAHs can mimic a dip at similar wavelengths. We find that the $9.7 \mu \mathrm{m}$ feature is probably present in all spectra.

We then compared the N-band spectra and nuclear 1-10 $\mu \mathrm{m}$ SEDs determined for our targets (Alonso-Herrero et al. 2003), to derive constraints on the obscuring material around the central engine. In the examples shown in Fig. 2 we find that the models provide a good description of the IR data for Mk573 and a satisfactory fit for NGC7674, where the photometric data is well matched by the model, but the $9.7 \mu \mathrm{m}$ dust feature is not well reproduced. The high inclination angle of the torus in NGC7674 is in good agreement with the Compton-Thick column obseved in X-rays (Bassani et al. 1999). 

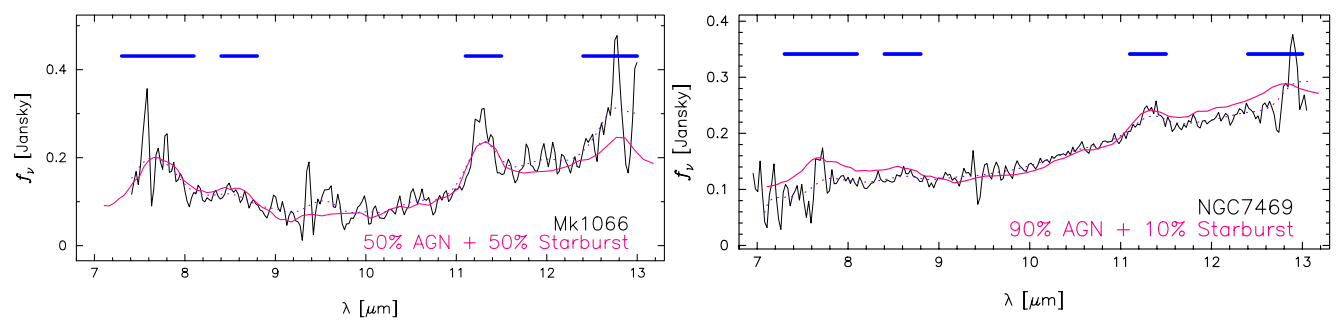

Figure 1. N-band Michelle spectra for 2 of the Seyfert galaxies in our sample (thin continuous line). The positions of the more prominent PAHs are shown. The spectra were smoothed (dashed line) for comparison with ISO templates of AGN + starburst emission in order to estimate the contribution to the data from star-forming regions (thick continuous line).
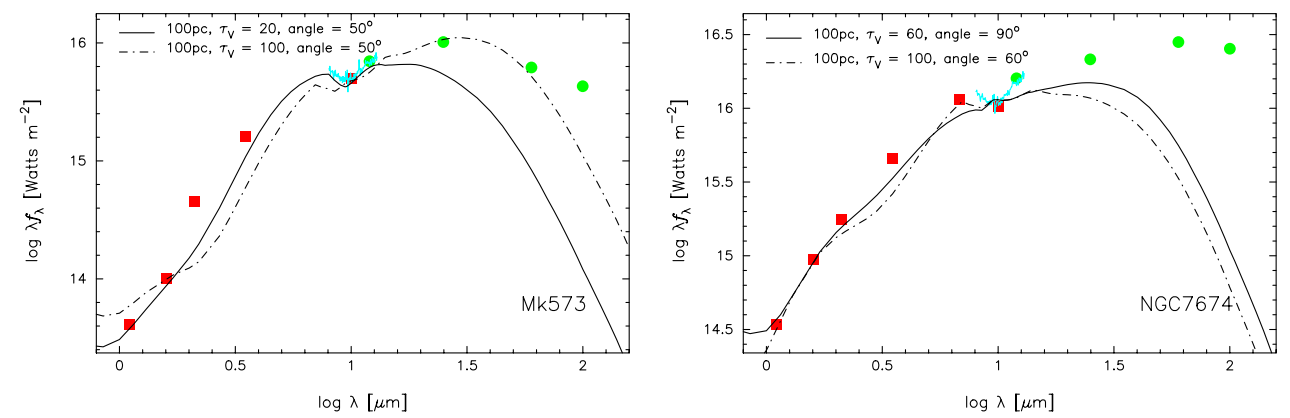

Figure 2. Nuclear SEDs (squares) and N-band Michelle spectroscopy compared to torus models (Nenkova, Ivezić \& Elitzur, 2002). IRAS data (circles), including emission from the whole galaxies, are also shown. Some of the model parameters are shown in the figures.

These preliminary results will be greatly improved once the larger database has been fully analysed. The observations and modelling will be used to: (a) Test whether the same overall torus geometry and physical conditions can explain the observed properties of all objects; (b) Constrain model parameters such as optical depth, geometry and extent of the torus; compare dust and X-ray columns for galaxies in common with Bassani et al. (1999); (c) Look for possible correlations between torus characteristics and other traits of the central source, such as luminosity, intermediate (ie, type S1.8 and S1.9) classification, the presence of polarised BLR or broad wings seen in the IR; (d) Study the circumnuclear emission to test a 'non-strict' Unified Model (Alonso-Herrero et al. 2003; Matt 2000) which suggests that the obscuration in some S2s might be due to extended material, as well as the possible connection between the lack of hidden BLRs in cool S2s and the presence of enhanced starburst emission (Alexander 2001).

\section{Acknowledgements}

Support for this work was provided by Fundación Andes.

\section{References}

Alexander, D. M. 2001, MNRAS, 320, L15

Alonso-Herrero, A., et al. 2003, AJ, 126, 81

Bassani, L., Dadina, M., Maiolino, R., Salvati, M., \& Zamorani, G. 1999, ApJS, 121, 473

Matt, G. 2000, A\&A, 355, L1

Nenkova, M., Ivezić, Z., \& Elitzur, M. 2002, ApJ, 570, L9

Rush, B., Malkan, M. A., \& Spinoglio, L. 1993, ApJS, 89, 1

Tran, H. D. 2003, ApJ, 583, 632 